\title{
Posición hioidea, posición lingual y dimensión de la vía aérea faríngea según maloclusión esquelética
}

\author{
Hyoid position, lingual position and dimension of the \\ pharyngeal airway according to skeletal malocclusion \\ Posição hióide, posição lingual e dimensão faríngea das vias \\ aéreas de acordo com a maloclusão esquelética \\ Miriam Janeth Espada De-La-Cruz' (D) 0000-0002-7681-3927 \\ Luciano Carlos Soldevilla Galarza² ${ }^{2}$ (D) 0000-0002-5939-4644 \\ Manuel Antonio Mattos-Vela ${ }^{3}$ (iD 0000-0002-5701-1961
}

\section{Resumen}

Esta investigación se centró en revisar los artículos que evaluaron cefalométricamente la posición de hueso hioides, posición lingual y la dimensión de la vía aérea faríngea según maloclusión esquelética, a fin de determinar si existe una relación entre estas estructuras. Método: Se identificaron publicaciones en las bases de datos: PubMed, Biblioteca Virtual en Salud (BVS), Scielo y Scopus. Palabras clave: Hueso hioides, Lengua, Vía aérea, Maloclusión. Se realizó un cribado de los artículos por título, resumen y texto completo, escritos en los idiomas inglés y español.

Resultados: Se encontraron 75 artículos; se eliminaron 30, por no tener una relación directa con el tema. Finalmente, se seleccionaron 45 artículos. Se concluye que aún no hay un consenso absoluto, sobre la relación existente entre: la posición del hueso hioides, la lengua y la dimensión de la vía aérea superior; según maloclusión esquelética.

Palabras clave: Hueso Hioides, Lengua, Vía Aérea y Maloclusión.

1 Universidad Nacional Mayor de San Marcos, Perú. miriam.espada@unmsm.edu.pe 


\section{Abstract}

This research focused on reviewing the articles that evaluated cephalometrically the position of the hyoid bone, lingual position and the dimension of the pharyngeal airway according to skeletal malocclusion, in order to determine if there is a relationship between these structures.

Method: Publications were identified in the following databases: PubMed, Virtual Health Library (VHL), Scielo and Scopus. Key words: Hyoid bone, Tongue, Airway, Malocclusion. Articles were screened by title, abstract and full text, written in English and Spanish.

Results: 75 articles were found, 30 were eliminated for not having a direct relation with the topic. Finally, 45 articles were selected. It is concluded that there is still no absolute consensus on the relationship between the position of the hyoid bone, the tongue and the dimension of the upper airway, according to skeletal malocclusion.

Keywords: Hyoid Bone, Tongue, Airway, and Malocclusion.

\section{Introducción}

El diagnóstico en la ortodoncia es fundamental para el tratamiento integral de las maloclusiones. Para realizarlo existen diversas técnicas y herramientas de diagnóstico: como radiografías, modelos de estudio, fotografías, entre otras.

El hueso hioides juega un papel muy importante en la masticación, fonación y deglución. También, ayuda a mantener las vías respiratorias sin obstrucciones, debido a la unión del músculo infrahioidal (omohioideo) a la cintura escapular. Además, este hueso está en estrecha relación con la lengua, ya que están unidos mediante el geniogloso y el músculo geniohioideo.

\section{Resumo}

Esta investigação centrou-se na revisão dos artigos que avaliaram cefalometricamente a posição do osso hióide, a posiçáo linguística e a dimensão da via aérea faríngea de acordo com a maloclusão esquelética, a fim de determinar se existe uma relação entre estes estruturas.

Método: As publicações foram identificadas nas seguintes bases de dados: PubMed, Biblioteca Virtual em Saúde (BVS), Scielo e Scopus. Palavras-chave: Osso hióide, Língua, Via aérea, Maloclusão. Os artigos foram analisados por título, resumo e texto completo, escritos em inglês e espanhol.

Resultados: Foram encontrados 75 artigos, 30 foram eliminados porque não estavam directamente relacionados com o tema. Finalmente, foram seleccionados 45 artigos. Conclui-se que ainda não existe consenso absoluto sobre a relação entre: a posição do osso hióide, a língua e a dimensão da via aérea superior, de acordo com a maloclusão esquelética.

Palavras-chave: Osso Hioide, Língua, Via Aérea, e Maloclusão.

El órgano lingual, por su parte, ocupa gran espacio en la cavidad bucal y realiza movimientos en diversas funciones orales como la deglución, el habla y la respiración ${ }^{(1)}$. Por tanto, el análisis de la posición hioidea y la posición lingual serían indicadores para el paso de la vía aérea faríngea. La vía aérea faríngea, conocida también como espacio nasofaríngeo, constituye una estructura compleja y multifunctional, que regula diferentes procesos del organismo. También coordina funciones respiratorias y de ventilación, así como funciones gástricas y de fonación ${ }^{(2)}$. 
McNamara ${ }^{(3)}$ acepta que existe una controversia en la relación entre la función respiratoria, morfología facial y oclusión, pues reconoció plenamente que existen factores ambientales que favorecen la alteración de la función respiratoria.

Según Moss ${ }^{(4)}$, los conceptos de matriz funcional y de crecimiento óseo se dan en respuesta al comportamiento funcional del macizo cráneofacial de un individuo. En este sentido, las alteraciones en la vía aérea y la postura de la lengua pueden afectar el crecimiento maxilofacial. Sin embargo, aún no hay suficientes investigaciones que den sustento al flujo del aire como algo determinante en el crecimiento y desarrollo craneofacial. La disminución de la permeabilidad de las vías aéreas altera la respiración y provoca cambios en el desarrollo cráneo dentofacial normal. Por ello, un buen diagnóstico y tratamiento temprano pueden prevenir, restablecer o corregir el crecimiento cráneo-maxilofacial en un individuo ${ }^{(5)}$.

El presente trabajo tiene como objetivo revisar los artículos que evaluaron cefalométricamente la posición de hueso hioides, posición lingual y la dimensión de la vía aérea faríngea según las maloclusiones esqueléticas, para saber si existe una relación entre estas tres estructuras en cada maloclusión esquelética.

\section{Revisión y método}

Se revisó la literatura que ha tratado el tema de esta investigación, a partir de una búsqueda bibliográfica. Se utilizaron las bases de datos de Medline, Lilacs, Scielo y Scopus, haciendo uso de las palabras clave: Hueso hioides, Lengua, Vía aérea y Maloclusión. Se seleccionaron aquellos artículos que contenían información sobre la posición hioidea, posición lingual y dimensión de la vía aérea faríngea en maloclusiones esqueléticas, cuyos datos fueron extraídos de radiografías cefalométricas y tomografías computarizada de haz cónico: Las investigaciones escogidas fueron escritos en los idiomas inglés y español, durante el periodo del 2010 al 2021. También se incluyeron artículos de mayor antigüedad por la relevancia de la información sobre el tema. Se realizó un cribado de los artículos que se eligieron en primera instancia, luego se revisaron los títulos y resúmenes de todos los artículos encontrados. En total se encontraron 75 artículos, de los cuales se eliminaron 30, por no tener una relación directa con el tema. Finalmente, se seleccionaron 45 artículos originales (Fig 1).

Fig. 1: Diagrama de flujo del proceso de identificación y selección de artículos

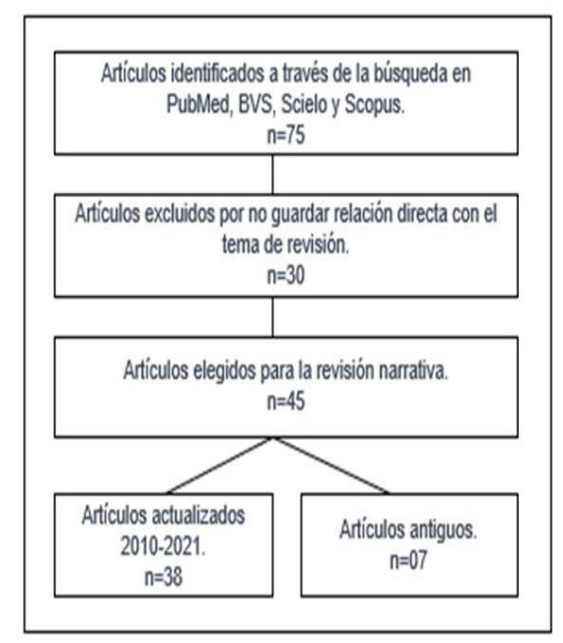

\section{a)Importancia del estudio de la posición hioidea, posición lingual y la vía aérea faríngea}

El análisis y la evaluación de la posición hioidea, posición lingual y la vía aérea faríngea en las maloclusiones han recibido una atención significativa en la literatura, debido a la necesidad de un diagnóstico completo y a la realización de un buen plan de tratamiento. Esto sugiere que el sistema estomatognático debe ser evaluado de manera integral y no parcial. La posición hioidea tiene un rol importante y activo en la realización del balance postural y permeabilidad de la vía aérea faríngea ${ }^{(6)}$. La capacidad de determinar con precisión la posición y volumen 
de la lengua es importante por dos razones. Primera, porque existe una estrecha relación con la posición del hueso hioides y su evaluación también determinaría el estado de la permeabilidad de la vía aérea faríngea; y segunda, porque permitirá evaluar la influencia del tamaño de la lengua en la morfología de la cara, las arcadas dentarias y en alguna alteración en su función oral (deglución, habla y respiración). Las dimensiones de la vía aérea faríngea constituyen un conocimiento fundamental para evaluar la apnea obstructiva del sueño (AOS) y para valorar los diferentes procedimientos quirúrgicos ortognáticos, debido al riesgo de obstrucción de las vías respiratorias ${ }^{(8)}$.

En la mayoría de los artículos estudiados, los investigadores hicieron análisis cefalométricos en radiografías laterales de cráneo; en otros, efectuaron el análisis cefalométrico en tomografías computarizadas de haz cónico.

\section{b) Relación de la posición hioidea, posición lingual y la dimensión de la vía aérea faríngea con la maloclusión esquelética}

La mayoría de los artículos encontrados relacionan por separado estas variables según la maloclusión esquelética [la posición del hueso hioides según la maloclusión esquelética ${ }^{(9-11)}$, posición de la lengua según la maloclusión esquelética $^{(12-17)}$ y la dimensión de la vía aérea faríngea según la maloclusión esquelética ${ }^{(15,18-26)}$ ] y solo una menor cantidad lo hace de manera conjunta [la posición del hueso hioides con la dimensión de la vía aérea faríngea según la maloclusión esquelética ${ }^{(5,7,28-31)}$, la posición del hueso hioides con la posición lingual según la maloclusión esquelética ${ }^{(32-36)}$, la posición lingual con la dimensión de la vía aérea faríngea según maloclusión esquelética ${ }^{(15)}$ y las evidencias de la relación entre el hueso hioides, posición lingual y la dimensión de la vía aérea faríngea según la maloclusión esquelética $\left.{ }^{(2,37-39)}\right]$.

Los artículos analizados mencionan que la ubicación del hueso hioides difiere entre las diferentes maloclusiones. Encontraron una ubica- ción inferior y anterior en la Clase I ${ }^{(9,11)}$; más inferior y posterior en la Clase II ${ }^{(2,8,9,11,31-38,41)}$; más anterior ${ }^{(11,30)}$ y superior ${ }^{(8,37,39)}$ en la Clase III. También, en pacientes de Clase III, se observa una inclinación inversa del hueso hioides (eje largo del hueso hioides no paralelo al plano mandibular) ${ }^{(10)}$. Otros estudios revelan una diferencia estadísticamente significativa en la posición e inclinación hacia el plano palatino y hacia la línea basión-nasión del hueso hioides en los pacientes de Clase I y III ${ }^{(10)}$.

Respecto de la postura lingual en reposo según la maloclusión esquelética, se encontró una ubicación más superior y posterior ${ }^{(12,13,16,37,38)}$ y un volumen más pequeño ${ }^{(37,39)}$ en la Clase II; más inferior $^{(12,14,38)}$, anterior ${ }^{(15,17)}$ y un volumen mayor ${ }^{(37,39)}$ en la Clase III ${ }^{(12-14,15,17)}$. También se descubrió que la posición lingual está asociada con las proporciones de las características dentales y alveolares del maxilar y la mandíbula ${ }^{(14)}$. Referente a la dimensión de la vía aérea faríngea según la maloclusión esquelética, se encontró que los pacientes de Clase II tiene una dimensión más pequeña (15,18,20-25,29-31,40), una orientación más hacia atrás y un volumen menor ${ }^{(8)}$. Los pacientes de Clase III tienen una dimensión más grande y un volumen mayor (6,15,18,21,24,30,31,40). Estas diferencias son estadísticamente significativas ${ }^{(20-23)}$.

Respecto de la relación entre la posición del hueso hioides con la posición lingual frente a las maloclusiones esqueléticas, varios artículos mencionan que sí existe una correlación. Los pacientes con maloclusión esquelética Clase II tienen relación con una posición más inferior y posterior del hueso hioides y una posición de lengua más superior, posterior y un volumen más pequeño. Los pacientes de Clase III tienen una relación con una posición más anterior y superior del hueso hioides y una posición lingual más inferior, anterior, y con un volumen mayor ${ }^{(33-36,45)}$. También se encontró que existe correlación entre la posición del hueso hioides con la posición lingual en personas con crecimiento vertical ${ }^{(32)}$. 
Referente a la relación entre la posición del hueso hioides con la dimensión de la vía aérea faríngea frente a las maloclusiones esqueléticas, en los artículos consultados se establece que en las maloclusiones Clase I existe una correlación positiva entre la dimensión de la vía aérea inferior y la distancia horizontal del hueso hioides al retrognation, ${ }^{(28)}$. Asimismo, la posición del hueso hioides se relaciona con la dimensión de la vía aérea faríngea ${ }^{(8,29)}$. Se encontró que la dimensión de la vía aérea es significativamente más estrecha y la ubicación del hueso hioides está más posterior e inferior en la Clase II ${ }^{(31)}$. También se menciona que cuanto más protuberante sea la mandíbula (SNB), más anterior será el hueso hioides y mayor será la dimensión de la vía aérea faríngea ${ }^{(32)}$.

Las evidencias de la relación entre el hueso hioides, posición lingual y la dimensión de la vía aérea faríngea en las maloclusiones indican que las Clases III tienen grandes volúmenes de lengua.

Respecto de la Clase II, se encontró que el equilibrio anatómico (proporcionalidad de volúmenes) entre la lengua y la cavidad oral fue mayor (38). Se descubrió, asimismo, que el ancho orofaríngeo superior era más estrecho y que el dorso de la lengua se ubica más superior en sujetos hiperdivergentes. El hueso hioides se ubica más inferior y posterior en sujetos hipodivergentes. Se observaron variaciones en el ancho de la orofaringe superior e inferior, la postura de la lengua y la posición del hueso hioides en todos los patrones de crecimiento ${ }^{(33,38)}$.

Se encontró una fuerte correlación entre las medidas en CBCT y cefalogramas laterales ${ }^{(7,43-45)}$.

\section{Discusión}

Casi todas las investigaciones consultadas utilizaron el ángulo ANB para establecer las relaciones anteroposteriores de la mandíbula; pero es necesario mencionar que este tiene limitaciones bien conocidas y que está influenciado por muchas variables como la morfología del área del nasion, las dimensiones verticales de la cara, la inclinación de la base craneal anterior y la inclinación de las mandíbulas. Si solo se utiliza el ángulo ANB para medir la posición relativa del maxilar y la mandíbula entre sí, la ubicación de los puntos A y B en el plano vertical influirá en el tamaño del ángulo y no en la relación sagital real de las mandíbulas. Sin embargo, el ángulo ANB todavía se reconoce como una forma tradicional de determinar las maloclusiones anteroposteriores y también fue aceptado en este trabajo.

Según la relación que existe entre cada estructura y la maloclusión esquelética, en esta revisión se descubrió que la posición del hueso hioides difiere entre las diferentes maloclusiones, según varios artículos ${ }^{(8,9,11,31-38,40)}$. Sin embargo, Nejaim et al. $^{(6)}$, encontraron que la posición del hueso hioides según sus dimensiones: anteroposterior, vertical y angular no presenta diferencias estadísticamente significativas, entre los tres tipos de maloclusiones esqueléticas. Por otra parte, José et al. ${ }^{(28)}$,en sus estudios evaluaron solo la posición del hueso hioides en la dimensión anteroposterior y también concluyeron que no presenta diferencias estadísticamente significativas entre las tres maloclusiones esqueléticas. Creemos que estas diferencias en los resultados se deben a la aplicación de diferentes metodologías de análisis cefalométrico y a la variación en las características de las muestras.

Para la postura lingual en reposo, se encontró que difiere según la maloclusión esquelética ${ }^{(2,12-}$ ${ }^{17,38)}$. Sin embargo, Fatima et al. ${ }^{(12)}$ concluyeron que no se encontraron diferencias significativas entre la postura lingual y las maloclusiones esqueléticas. Estos resultados sugirieron que, aunque no se encontró una diferencia estadísticamente significativa, puede haber variaciones de moderadas a grandes en la relación esquelética sagital con diferentes posturas de la lengua. Por tanto, se debe tener una inferencia clínica, monitorear la postura lingual durante el tratamiento de ortodoncia interceptivo e integral 
para poder tener una mayor estabilidad a largo plazo en los resultados obtenidos.

Respecto de la dimensión de la vía aérea faríngea según la maloclusión esquelética, se encontraron diferencias que fueron estadísticamente significativas en varios estudios ${ }^{(5,6,8,15,18,20,21,23,24,30-32)}$. Al respecto, Kaur et al. ${ }^{(44)}$ compararon estadísticamente los tres grupos de ANB y descubrieron una diferencia significativa para el volumen de la vía aérea faríngea. Concluyeron también que la disminución de la dimensión de la vía aérea faríngea en pacientes de Clase II se debe a la diferente ubicación de la lengua y la mandíbula; y el aumento de la dimensión de la vía aérea faríngea en la Clase III se debe a la posición anterior de la mandíbula y la posición de la lengua más baja. Sin embargo, Clokotiya et al. ${ }^{(26)}$ concluyeron que las dimensiones de la vía aérea faríngea no son afectadas por los cambios del ángulo ANB.

Según la relación conjunta que existe entre estas estructuras y la maloclusión esquelética, varios estudios anteriores determinaron que existe relación entre la posición del hueso hioides y la posición lingual según la maloclusión esquelética; encontraron que la posición del hueso hioides antes y durante los tratamientos ortodóncicos nos orienta en la posición y función de la lengua, ${ }^{(29,34-37)}$ debido a un anterior desplazamiento del hueso hioides, dando lugar a cambios en los movimientos de la lengua. Sin embargo, Seok ${ }^{(33)}$ encontró que la relación de la posición del hueso hioides y la posición lingual según la maloclusión esquelética no fue estadísticamente significativa; pero sí descubrió una correlación positiva entre los pacientes con crecimiento vertical. En las investigaciones consultadas, refieren también la existencia de una relación funcional entre la lengua, la mandíbula y el hueso hioides, representada por las conexiones musculares existentes entre estas partes, como son el geniogloso, constrictor faríngeo, geniohioideo, milohioideo y los dos músculos longitudinales. Estas interrelaciones deben tenerse en cuenta durante el diagnóstico clínico y el tratamiento ${ }^{(28)}$.
Shokri et al. ${ }^{(8)}$ y Ulusoy et al. ${ }^{(29)}$ encontraron que la posición del hueso hioides tiene una relación directa con las dimensiones de la vía aérea faríngea y debe considerarse durante la cirugía ortognática, debido al riesgo de obstrucción de las vías respiratorias. Sin embargo, Ramos et al. ${ }^{(30)}$ concluyeron que la posición hioidea es muy variable, por lo cual fue imposible determinar con exactitud dichas dimensiones. Reconocieron, sin embargo, que no existe suficiente evidencia para afirmar si existe una correlación estadísticamente significativa entre el hueso hioides y la dimensión de la vía aérea faríngea.

Iwasaki et al. ${ }^{(2)}$, Nejaim et al. ${ }^{(6)}$, Shokri et al. ${ }^{(8)}$, Lakshmi et al. ${ }^{(19)}$, Tianhu et al. ${ }^{(20)}$, Mendoza et al. ${ }^{(21)}$, Silva et al. ${ }^{(22)}$, Lopatiene et al. ${ }^{(23)}$, Zadzeviciute et al. ${ }^{(31)}$, Tarkar et al. ${ }^{(39)}$ encontraron diferencias significativas en la posición del hueso hioides, la posición lingual y la dimensión de la vía aérea faríngea en las tres maloclusiones esqueléticas. Sin embargo, Chauhan et al. ${ }^{(39)}$ no descubrieron diferencias entre las medidas angulares del hueso hioides y las maloclusiones de Clase I y Clase II división I. Ellos evaluaron la relación que tienen la dimensión de la vía aérea faríngea la posición de la lengua y el hueso hioides en sujetos con diferentes patrones dentofaciales, y concluyeron que no hubo diferencias ni en la dimensión anteroposterior de la vía aérea faríngea ni en la posición y relación del hueso hioides y la lengua, entre los pacientes con maloclusiones de Clase I y Clase II división I. Esos resultados podrían deberse al hecho de que sólo se tuvieron en cuenta las dimensiones anteroposteriores. Los puntos de referencia anatómicos y la subdivisión de las regiones anatómicas de la vía aérea faríngea utilizados en este estudio fueron diferentes de los recomendados y, por lo tanto, surge otro obstáculo importante para la comparación total con la mayoría de estos estudios. La falta de una definición subregional precisa de la vía aérea faríngea en la literatura limita su evaluación, lo que dificulta la comparación entre los estudios.

Liégeois et al. ${ }^{(7)}$ encontraron una fuerte correlación entre las medidas obtenidas en tomografías 
computarizadas de haz cónico y cefalogramas laterales. Kaur et al. ${ }^{(43)}$ concluyeron, también, que las mediciones de ambas modalidades son confiables y reproducibles, pero las radiografías cefalométricas laterales no brindan información sobre las mediciones volumétricas de la vía aérea superior. Confirmaron que el volumen de la vía aérea por TC muestra más variabilidad que la vía aérea correspondiente al área. Abramson et al. ${ }^{(44)}$ correlacionaron los hallazgos de la tomografía computarizada tridimensional entre las dimensiones de las vías respiratorias y las mediciones cefalométricas laterales. Los resultados arrojaron que la tomografía computarizada tridimensional y las mediciones cefalométricas laterales son fiables y reproducibles. Vizzotto et al. ${ }^{(45)}$ evaluaron la precisión de las mediciones de las vías respiratorias, a partir de cefalogramas laterales, reconstrucciones laterales CBCT y planos axiales CBCT; y demostraron que las mediciones lineales de las vías respiratorias son fiables, tanto con cefalogramas laterales como con reconstrucción CBCT.

\section{Conclusiones}

No existe un consenso absoluto sobre la relación existente entre: la posición del hueso hioides, la lengua y la dimensión de la vía aérea superior; según las maloclusiones esqueléticas, en los artículos consultados en esta revisión.

Las mediciones cefalométricas y las realizadas mediante tomografías computarizadas de haz cónico son confiables y reproducibles, con la diferencia de que estas últimas brindan además una información sobre medidas transversales y volumétricas.

Se debe evaluar, relacionar y valorar el sistema estomatognático, de manera integral y no parcial. Así mismo, se deben estandarizar las técnicas de análisis cefalométrico y las subdivisiones anatómicas regionales referente a la vía aérea faríngea. De este modo se facilitará la comparación de resultados entre las futuras investigaciones.

\section{Referencias}

1. Proffit WR. Ortodoncia contemporánea. 4ta Ed. España: Editorial Elsevier; 2008.

2. Iwasaki T, Suga H, Minami A, Sato H, Hashiguchi M, Tsujii T. Ralationships among tongue volumen, hyoid position, airway volumen, and maxilofacial form in pediatric patients with Class-II, and ClassIII malocclusions. Orthod Craniofac. 2019;22(1):9-15.

3. McNamara JA. Influence of respiratory pattern on craniofacial growth. Angle Orthod. 1981;51:269-300.

4. Moss M. The primary role of functional matrices in facial growth. Am J Orthod. 1959;55:566-567.

5. Kaur S, Rai S, Kaur M. Comparación de la confiabilidad del cefalograma lateral y la tomografía computarizada para la evaluación del espacio de las vías respiratorias. Niger J Clin Pract. 2014;17:629-36.

6. Nejaim Y, Johan K, Groppo F, Neto F. Evaluation of pharyngeal space and its correlation with mandible and hyoid bone in patients with different skeletal classes and facial types. American Journal of Orthodontics and Dentofacial Orthopedics. 2018;153(6):825-833.

7. Liégeois F, Albert A, Limme M. Comparison between tongue volume from magnetic resonance images and tongue area from profile cephalograms.Eur J Orthod. 2010; 32(4): 381-6.

8. Shokri A, Mollabashi V, Zahedi F, Tapak L. Position of the hyoid bone and its correlation with airway dimensions in different classes of skeletal malocclusion using cone-beam computed tomography. Imaging Science in Dentistry. 2020;50:105-15.

9. Mortazavi S, Asghari H, Dehghani M, Aboutorabzade M, Yaloodbardan B, Tohidi E. Hyoid bone position in different facial skeletal patterns. J Clin Exp Dent. 2018;10(4):346-51.

10. Adamidis L, Meropi N. Hyoid bone position and orientation in Class I and Class III maloclusions. Am J Orthod Dentofac, 1992;101:308-12.

11. Seher A, Neval D, Jalen k. Cephalometric Investigation of First Cervical Vertebrae Morphology and Hyoid Position in Young Adults with Different Sagittal Skeletal Patterns. Sci World J. 2014;1:1-8. 
12. Fatima F, Mubassar F. The assessment of resting tongue posture in different sagittal skeletal patterns. Dental Press J Orthod. 2019;24(3):55-63.

13. Verma S, Tandon P, Agrawal D, Prabhat K. A cephalometric evaluation of tongue from the rest position to centric occlusion in the subjects with class II division 1 malocclusion and class I normal occlusion. J Orthod Sci. 2012; 1 (2): 34-9

14. Primozic J, Farcnik F, Perinetti G, Richmond S, Ovsenik M. The association of tongue posture with the dentoalveolar maxillary and mandibular morphology in Class III malocclusion: a controlled study. Eur J Orthod. Junio de 2013; 35 (3): 388-93.

15. Iwasaki T, Sato H, Suga H, Takemoto Y, Inada E, Saitoh I, et al. Relationships among nasal resistance, adenoids, tonsils, and tongue posture and maxillofacial form in Class II and Class III children. Am J Orthod Dentofacial Orthop. 2017;151:929-40.

16. Yılmaz F, Sağdıç D, Karaçay S, Akin E, Bulakbası N. Tongue movements in patients with skeletal Class II malocclusion evaluated with real-time balanced turbo field echo cine magnetic resonance imaging. Am J Orthod Dentofacial Orthop. 2011;139(5):e415-25.

17. Gorgülü S, Sagdic D, Akin E, Karacay S, Bulakbasi N. Tongue movements in patients with skeletal Class III malocclusions evaluated with real-time balanced turbo field echo cine magnetic resonance imaging. Am J Orthod Dentofacial Orthop. 2011; 139 (5): 405-14.

18. Oh K, Hong J, Kim Y, Cevidanes L, Ho P. Three-dimensional analysis of pharyngeal airway form in children with anteroposterior facial patterns. Ortod de ángulo. 2011; 81 (6): 1075-82.

19. Lakshmi K, Yelchuru S, Chandrika V, Lakshmikar O, Sagar V, Reddy G. Comparison between growth patterns and pharyngeal widths in different skeletal malocclusions in South Indian Population. J Int Soc Prevent Communit Dent. 2018;8:224-8.

20. Wang T, Yang Z, Yang F, Zhang M, Zhao J, Chen J, et al. A Three Dimensional Study of Upper Airway in Adult Skeletal Class II Patients with Different Vertical Growth Patterns. PLoS ONE. 2014; 9(4):955-44.

21. Mendoza J. Comparación de la dimensión del espacio aéreo faríngeo según las deformidades esqueléticas Clase I, II y III en radiografías cefalométricas de pacientes que asistieron a la clínica docente UPC entre los años 2011 al 2014 [Pregrado]. Universidad Peruana de Ciencias Aplicadas;2017.

22. Silva N, Lacerda R, Silva A, Ramos T. Assessment of upper airways measurements in patients with mandibular skeletal Class II malocclusion. Dental Press J Orthod. 2015;20(5):86-93.

23. Lopatiené K, Sidlauskas A, Vasiliauskas A, Cecyte L, Svalkauskiene V, Sidlauskas M. Relationship between malocclusion, soft tissue profile, and pharyngeal airways: A cephalometric study. Medicina (Kaunas). 2016;52(5):307-314.

24. Zheng ZH, Yamaguchi T, Kurihara A, Li HF, Maki K. Three-dimensional evaluation of upper airway in patients with different anteroposterior skeletal patterns. Orthod Craniofac Res. 2014;17(1):38-48.

25. Claudino LV, Mattos CT, Ruellas AC, Sant' Anna EF. Pharyngeal airway characterization in adolescents related to facial skeletal pattern: a preliminary study. Am J Orthod Dentofacial Orthop. 2013;143(6):799-809.

26. Chokotiya H, Banthia A, K SR, Choudhary K, Sharma P, Awasthi N. A Study on the Evaluation of Pharyngeal Size in Different Skeletal Patterns: A Radiographic Study. J Contemp Dent Pract. 2018;19(10):1278-1283.

27. Messina. The tongue, mandible, hyoid system. Eur J Transl Myol. 2017; 27(1): 74-76

28. Jose N, Shetty S, Mogra S, Shetty V, Rangarajan S, Mary L. Evaluation of hyoid bone position and its correlation with pharyngeal airway space in different types of skeletal malocclusion. Contemp Clin Dent. 2014;5(2):187-9.

29. Ulusoy C, Canigur Bavbek N, Tuncer BB, Tuncer C, Turkoz C et al. Evaluation of airway dimensions and changes in hyoid bone position following class II functional therapy with activator. Acta Odontol Scand. 2014; 72 (8): 917-925.

30. Ramos M, Morales R, Samanamú S, Gómez A, Alva C. Posición del hueso hioides en relación al volumen de la vía aérea en los diferentes patrones esqueletales. Kiru. 2018;1(3):106-112. 
31. Sadzeviciute E, Nazimova J, Trakiniene G. The impacto $f$ the hyoid bone position on the pharyngeal airway characteristic among different facial skeletal patterns. Stomatologija. 2019; 21(4):99-106.

32. Cheng J, Hsiao S, Chen C, Hsu K. Relationship between hyoid bone and pharyngeal airway in different skeletal patterns. J Dent Sci. 2020;15(3):286-293.

33. Seok J. Correlation análisis between tongue posture and hyoid bone position and cephalometric análisis variables in adult female patients. Korea J Orthod.2014;30(5):579-89.

34. Tallgren A, Solow B. Hyoid bone position, facial morphology and head posture in adults.Eur JOrthod.1987;9:1-8.

35. Haralabakis N, Toutountzakis N, Yiagtzis S. The position of the hyoid bone in adult individuals with an open bite and normal occlusion. Eur J Orthod.1993;15:265-271.

36. Subtelny J, Sakuda M. Open bite: diagnosis and treatment. Soy J Orthod.1964;50:337-358.

37. Castrillo A, Alonso M. Perez L, Colome G, Alaloya C, Medina S. Biotipo facial y posición hioidea en pacientes que inician tratamiento ortodóncico. Revista ADM. 2016;73(6):297-302.

38. Tarkar J, Parashar S, Gupta G, Bhardwaj P, Maurya R, Singh A et al. An evaluation of upper and lower pharyngeal airway width, tongue posture and hyoid bone position in subjects with different growth patterns. J Clin Diagn. 2016;10(1):79-83.

39. Chauhan A, Autar R, Lata K, Yadav V. Comparison of pharyngeal airway dimensión, tongue and hoid bone position base don ANB angle. Natl Maxillofac Surg. 2015;6(1):42-51.

40. Zou Y, Fu QM, Xu XY. Relationships among tongue volume, hyoid position, airway volume and maxillofacial form in pediatric patients with Class I, Class II and Class III malocclusions. Shanghai Kou Qiang Yi Xue. 2020;29(6):632-637.

41. Tseng Y, Tsai F, Chou S, Hsu C, Cheng J, Chen C. Evaluation of pharyngeal airway volume for different dentofacial skeletal patterns using cone-beam computed tomography. J Dent Sci. 2021;16(1):5157.

42. Khanna R, Tikku T, Sharma VP. Posición y orientación del hueso hioides en materias de clase II división 1: un estudio cefalométrico. J Ind Orthod Soc. 2011; 45 (4): 212-218.

43. Kaur S, Rai S, Kaur M. Comparación de la confiabilidad del cefalograma lateral y la tomografía computarizada para la evaluación de las vías respiratorias. Niger J Clin Pract.2014;17:629-36.

44. Abramson ZR, Susarla S, Tagoni JR, Kaban L. Análisis tomográfico computarizado tridimensional de la anatomía de las vías respiratorias. J Oral Maxillofac Surg 2010; 68: 363-71.

45. Vizzotto MB, Liedke GS, Delamare EL, Silveira HD, Dutra V, Silveira HE. Un estudio comparativo de cefalogramas laterales e imágenes de tomografía computarizada de haz cónico en la evaluación de la vía aérea superior. Euro J de Ortodoncia 2011; 34: 390-3.

\section{Nota contribución de los autores:}

1. Concepción y diseño del estudio

2. Adquisición de datos

3. Análisis de datos

4. Discusión de los resultados

5. Redacción del manuscrito

6. Aprobación de la versión final del manuscrito

MJEDLC ha contribuido en 1, 2, 3, 4, 5 y 6 .

LCSG ha contribuido en 1 y 6.

MAMV ha contribuido en 5 y 6.

\section{Nota de aceptación:}

Este artículo fue aprobado por la editora de la revista Mag. Dra. Vanesa Pereira-Prado. 